\title{
The Impact of Formal Properties on Eye Movements during the Perception of Architecture
}

\author{
RALF WEBER, YUN CHOI, and LAWRENCE STARK \\ University of California, Berkeley, USA \\ Technical University of Dresden, Germany
}

\begin{abstract}
The project represented an initial attempt to record how the visual experience of architecture is influenced by various formal-geometric characteristics such as size, contrast, direction, symmetry, closure, etc., and how these factors may alter individual visual scanpaths and affect awareness and appreciation of architectural designs. Results indicate that the eye does not trace shapes completely, but focuses on the overall arrangement of visual centers, major masses, and on objects with distinct formal differences from the overall set. Elements indicating spatial depth such as vistas receive special attention. There is a clear preference for the left area of a space. Redundant elements draw less attention than solitary shapes. Vertically and horizontally oriented objects are explored less than obliquely oriented shapes. More work using the methods of this study might provide important findings in the following three areas: scientific analysis of certain characteristic properties of architectural form and space long described by writers on architectural aesthetics; exploration of discrepancies in the perception of two-dimensional and three-dimensional representations of architectural form and space in the design process; and an evaluation of differences in the perception of architectural form and space by architects and non-architects.
\end{abstract}

\section{INTRODUCTION}

Much of the way the built environment is conceived and designed by architects rests on assumptions about how particular geometric arrangements are perceived by the human eye. Architects like to postulate that they can influence the sequence of eye movements during the perception of buildings through design decisions and, therefore, draw attention to particular architectural elements. Yet most of these suppositions about the visual perception of buildings rest on pure speculation, on 'common sense', and on traditions of aesthetic theories of visual harmony. Rarely do they have scientific basis.

How do people look at buildings and architectural space? Is the way the human eye 'looks' at buildings and spaces individually determined by cognitive models? Or are there particular physical, geometric variables that determine eye movements? What elements of architectural form trigger the attention of the eye more strongly than others? How are spaces perceived when architectural elements are altered or replaced? Questions of this type have occupied the profession for centuries. Through history, many now-classic treatises about architecture have speculated about the visual impact of form. And during this century, writers such as Rudolf Arnheim (Art and Visual Perception, The Dynamics of Architectural Form) ${ }^{\prime}$ have continued these efforts to evaluate the effect of form on the experience of architecture.

With the introduction of modern visual-analysis equipment new steps can be taken in this area of research. This equipment now makes it possible to describe precisely how the eye sees, and to measure the eye movements of people looking at buildings and interior architectural spaces. Scientific evidence of this kind might be used to test architects' claims that a viewer's eyes can be guided through an arrangement of spaces, masses and shapes. Major findings in this area could be particularly useful in the teaching of design, since there is currently a great absence of scientific basis for explanations of how form and space are experienced. In the long term, developing a more precise knowledge of how particular spatial features influence the perception of buildings may allow architects to think about design in a more rational, conscious manner.

This topic provides a considerable agenda for research in the coming years. The current study attempts only to make an initial investigation of the powers of modern visualanalysis equipment in relation to architectural space. In particular, it attempts to establish some initial principles explaining what variables influence areas of eye fixation and the visual scanpaths of people perceiving interior architectural spaces. Its principal results are two series of images. One depicts sequences of eye movements by individual subjects in relation to set compositions of architectural forms and spaces. The other depicts aggregate durations of eye fixations by a group of subjects in relation to the same compositions. 


\section{RELATED STUDIES IN \\ EYE MOVEMENT AND AESTHETICS}

Detailed visual information can only be obtained via the fovea, the small - about half a degree - central area of the retina that has the highest density of cone photoreceptors. The brain directs the eyes to move with fast saccades (jumping movements of the eye) to fixate (keep the eyes relatively stationary) and also to foveate (to place the fovea) successively onto the points of interest. Hence, eye movements (EMs) are necessary for scenes that span more than a few degrees in the subject's field of view. While viewing a stationary scene, the eyes alternate between fixations and saccades, during which vision is suppressed. Since fixations represent almost all of the time when visual information is collected, viewing can be characterized by the location and sequence of fixations.

The application of oculomotor recording to the field of architecture has hitherto been unexplored; indeed, there has been little research in the perception of three-dimensional form to this date. Studies of eye movement have traditionally dealt with normal reading, reading disabilities, visual tracking and scanning, and many other aspects of vision. Early pioneering work in eye movement that explicitly focused on the aesthetic impact of the display was performed by Buswell $(1935)^{2}$, using photographs of artworks, patterns and sculpture; and Brandt $(1945)^{3}$ who concentrated more particularly on the layout patterns of advertisements. Studies which emphasize the influences of formal characteristics in visual patterns, especially painting, have been undertaken by Kaufman and Richards (1969) ${ }^{4}$ and Molnar (1981) ${ }^{5}$. Kaufman and Richards examined the relative popularity of various parts of figures as resting places for the eye, measured by the relative amount of time spent there. They found the center of gravity is attractive, as are also the edges and corners; similar findings have been presented by Levy-Schoen ${ }^{6}(1973)$. Gould and Peeples ${ }^{7}$ (1973) claim that a subject's interpretation of a simple figure has no effect on the EMs he makes when viewing it, and that it is only its 'physical attributes' that determine the movements. By contrast, Noton and Stark's $(1971)^{8}$ studies pertain to the physiological and cognitive foundations of eye movement with a particular focus on scanpath patterns. The scanpath has been defined as a repetitive sequence of saccades and fixations, idiosyncratic to the viewer and to the picture. Storing and retrieving memories are important components of visual learning and pattern recognition.

\section{PROJECT DESCRIPTION}

The project was carried out at the Department of Architecture and the Telerobotics and Neurology Unit of the School of Optometry at the University of California at Berkeley. Viewers were presented for ten seconds each with ten models of architectural interior spaces and their photographs respectively. Seven seconds were devoted to observation of the image, and three seconds for verification of the calibration of the apparatus. Each test consisted of a random series of different views, featuring different compositions of typical architectural spatial situations.

The principal interior model consisted of a cube of $30 \mathrm{~cm}$ dimension with replaceable walls to allow changes in floor pattern and the opening of a background vista to a landscape. Variable elements used to furnish the interior of the box were a staircase, two columns, and three boxes to represent furniture. These interior spaces were arranged in ten different compositional layouts, each representing a typical spatial situation. One version showed an empty space; all others were arranged in pairs as a way to highlight the effect of certain particular geometric characteristics of architectural form and space. The following six characteristic properties were chosen for the test series: symmetry; left and right reversed; obliquely vs. rectilinearly oriented elements; foreground compared with middle ground and background vista; and foreground elements with and without a vista. The models and their two dimensional representations were seen from a 30 degree visual angle.

The twenty subjects were undergraduate students from the University of California, half from the Architecture Department and half from the School of Optometry. Each subject was presented with two sequences of the ten layouts

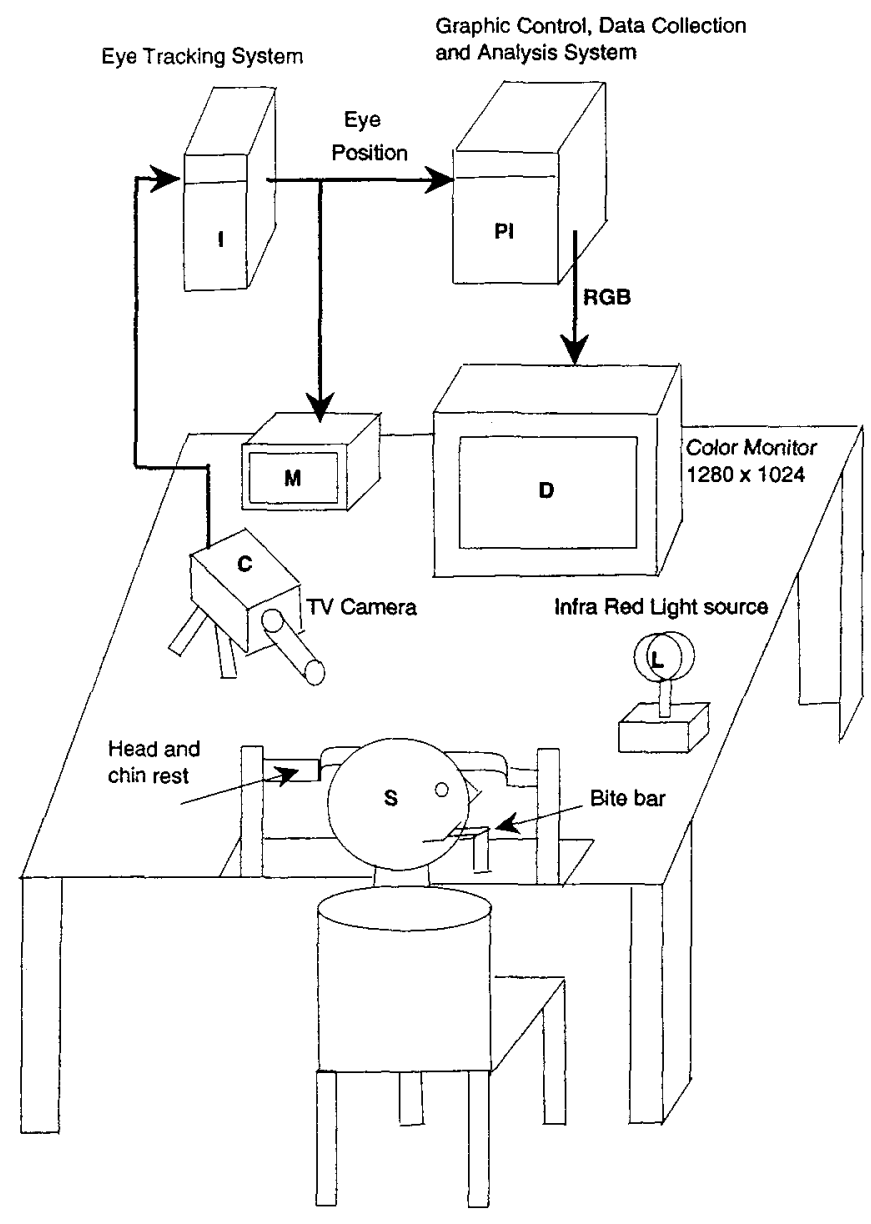

Fig. 1. Apparatus: Eye Tracking System 


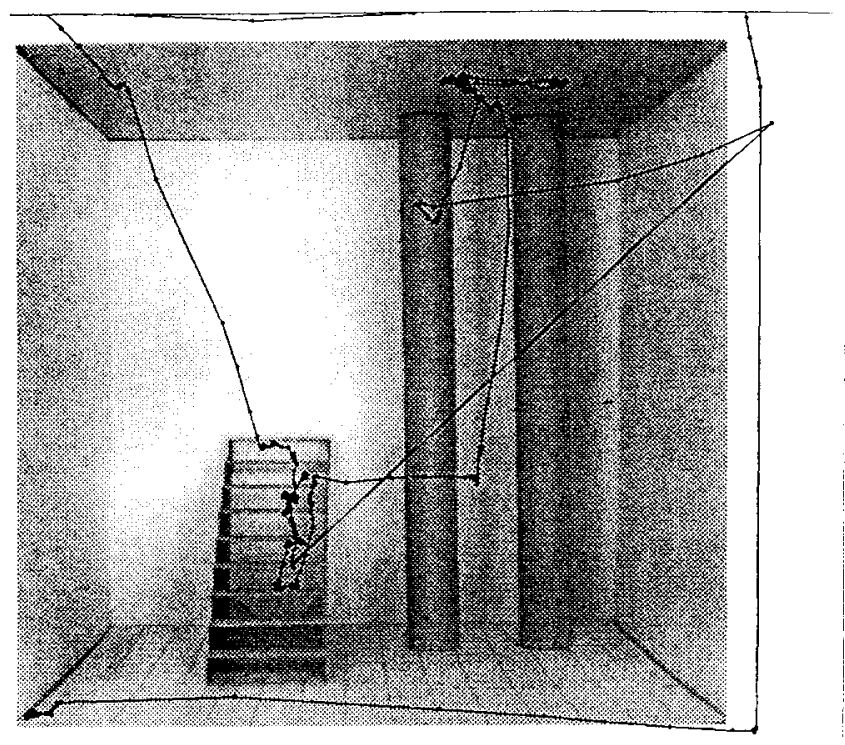

Fig. 2. Sample of individual Scanpath superimposed over viewed image

in random order. The images were shown for seven seconds each. After each image was shown, the subject looked at five dots in the corners and center of the screen to recalibrate. No specific tasks were given to the subjects; they were simply asked to look at the images. The principal data for analysis consisted of individual fixation scanpaths, that is, the order and duration of a subject's eye-fixation areas. These were carefully monitored using the apparatus diagrammed and described below.

The eye-movement (EM) measurement apparatus was a video-based system that included an infrared light source (L); a video camera with infrared filter (C) to capture the first Purkinje image (anterior surface reflection of the cornea); a bite-bar mounted on a chin- and headrest to abate head movements; a video monitor (M); software eye-tracking programs that accessed a frame grabber controlled by a Personal Iris (I) Computer; and a Power Series Silicon Graphics Iris Workstation 4D/120GTXB (PI), with two 16MHz RISC processors providing 20 MIPS and 2 MFLOPS to control image processing, data acquisition, and also to control picture display (D) to subjects (S). The sampling frequency was about $50 \mathrm{~Hz}$ (time-stamped so as not to lose temporal information due to the UNIX operating system). The accuracy of the EM measurement was a quarter of a degree vertically as well as horizontally.

The EM software was designed and written in the Telerobotics \& Neurology Unit at the School of Optometry at U.C. Berkeley by G. Tharp, H.S. Hsiung, M.J. Lipney, and Y.S. Choi. It included modules to perform image processing, socket communication, calibration, linearization, and the fixation-detection algorithm . The image-processing program calculated both the centers of the pupils and corneal light reflection based on images sampled from the camera by the frame grabber. Positional information was sent to the Power Iris through a UNIX socket; sockets also provided communication between programs running on different machines. Instantaneous EM measurement is a complex and time-consuming task; the study used two computers to provide more computational power to increase the processing speed, as well as simplify implementation of the simultaneous stimulus display and EM data collection tasks.

The experiment began by presenting a subject with the calibration procedure. The subject had to follow and fixate on the nine calibration dots (a 3 by 3 matrix), moving from left to right (12 degrees), and from the bottom up ( 9 degrees), on a 1,280-by-1,024 pixel screen. The initial calibration took approximately six seconds to complete. After this, the stimulus was presented-either a three-dimensional model or scanned photos. The stimuli were presented $-\mathrm{cm}$ away from the subjects, so that a $30 \times 30 \mathrm{~cm}$ Model will represent - a degree visual angle. Upon completion of viewing, linearized EM data was superimposed on the image for visual inspection. Fixation points were automatically found from linearized EM data, plotted on the image, and then connected in succession with straight lines to illustrate the scanpath.

\section{RESULTS AND OBSERVATIONS}

Results and observations from the tests at this point pertain only to an analysis of sequences and duration of eye fixations. The following discussion is based on the cumulative analysis of zones of fixation and the duration and sequence of fixations by all subjects in the test. The accompanying diagrams show which areas of the images presented to the subjects were scanned by the eye, how long fixations lasted, and in what sequence fixations occurred.

- An empty space: Most initial fixations occurred in the left half of the space. The primary area of attention was the middle of the space, while comparatively little attention was placed at the corners of the space. The spatial contours were typically not traced by the eye.

- Symmetry vs. asymmetry: In the symmetrical arrangement, the center element (stair) and the left column were the preferred targets of initial fixations. The right column was typically not an initial target, probably because of the redundancy of the columns. Also of interest is that the upper and lower parts of elongated forms will form visual centers, while the central part will typically not attract attention. In the asymmetrical configuration, both the stair and the columns drew attention during initial fixations. Attention was placed at the interstice between the columns. This may indicate that within a local pattern of symmetry (the two columns) the axis forms the center of attention. It is of further interest that in the case of the symmetrical arrangement, the viewer usually placed less attention on details. This would seem to indicate that redundancy of elements will reduce attention to the individual feature.

- Left and right reversed: If the primary visual element (in this case, the column) was placed on the left, it drew a 

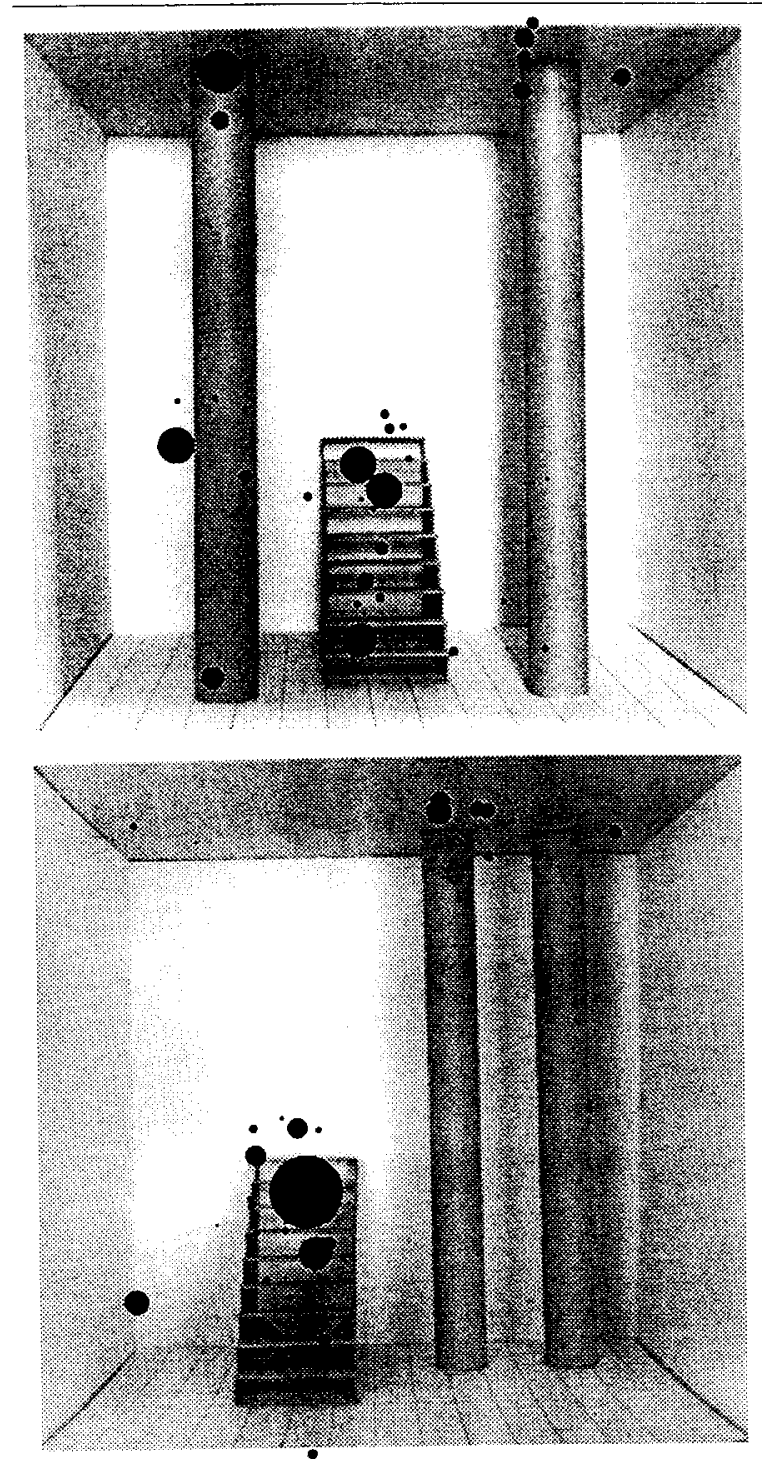

Fig. 3 \& 4: Symmetry vs. asymmetry. Cumulative Analysis showing duration of fixation by all non-architects in different areas of the composition. Clustered Scanpath showing sequence and duration of one architect's eye fixations.

greater number of initial fixations than it did when it was placed on the right. This is a significant finding in relation to the statements by Amheim that the left side of a composition attracts more attention than the right side. Apart from the differences in initial fixation, there were few differences in regard to the distribution of subsequent fixations between the two versions during the first six seconds.

- Obliquely oriented elements: In the alternative in which the stair was frontally presented, it seems to have been perceived as a whole; i.e., the individual steps were not scanned through individual fixations. In the side view, however, the steps were traced by individual fixations. In both initial as well as subsequent fixations, the oblique elements received most of the attention. In the first viewing, the steps were traced individually; in the second,

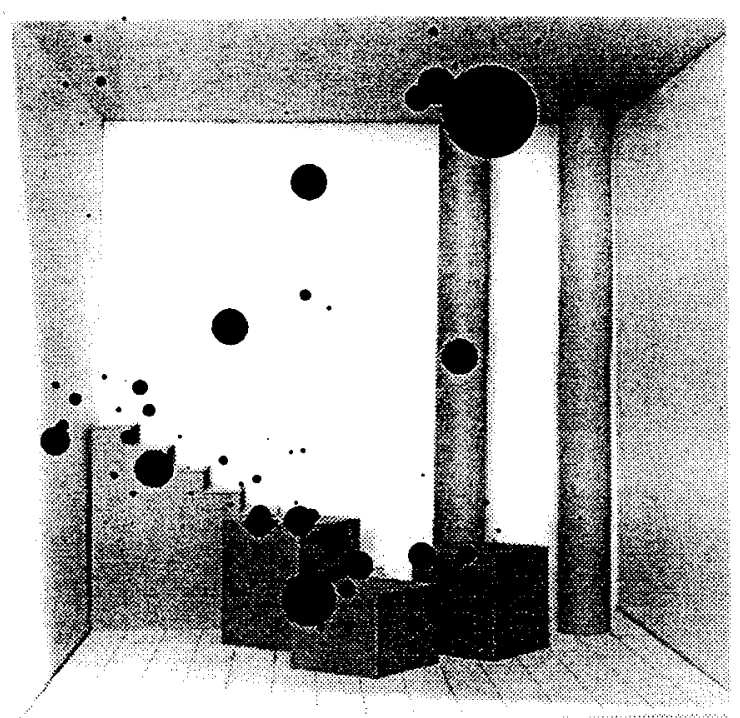

Fig. 5: Oblique Elements, Non-architects

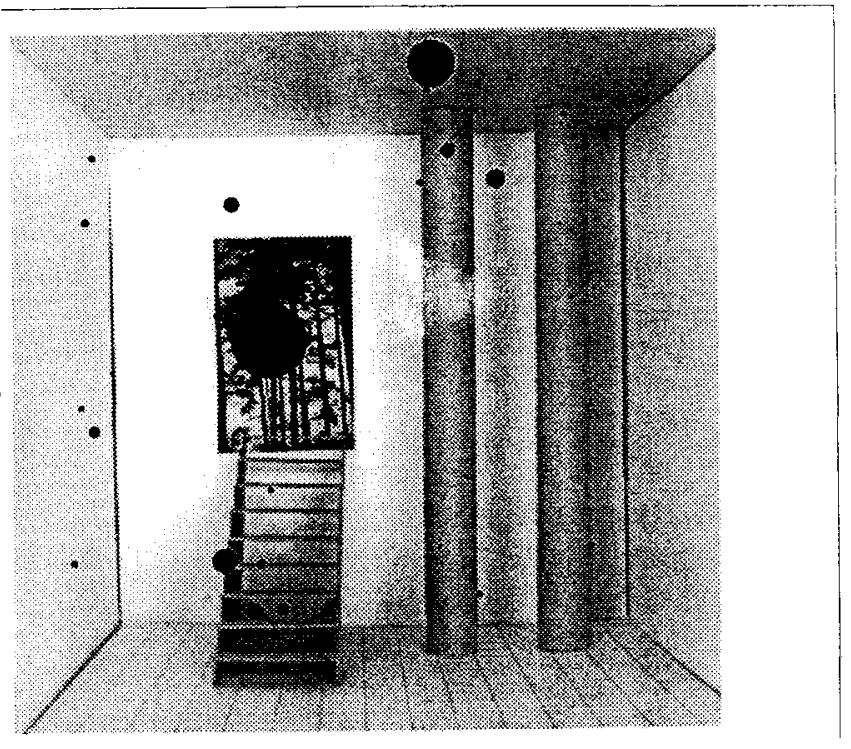

Fig. 6: Vista, non-architects

the shape of the stairs was viewed as a whole. This may indicate a learning process, i.e., the establishment of cognitive models which may influence the perception of previously known situations, as discussed by Noton and Stark'.

- Vista: The vista in the back wall was the principal locus of attraction for the initial fixations; other elements could not compete. Even for the other main fixations, the vista still formed the primary focus. The stairs and the columns formed secondary centers of attraction.

- Foreground, furnished, with and without vista: In the alternative without the vista, initial fixations were distributed all over the furnishing elements in the foreground, even though a slight dominance of the lower half of the image occurred, perhaps because of the attraction of the elements in the foreground. In the view with a vista, initial 
fixations were mainly concentrated in the area of the vista. As for the other main fixations, the foreground and the background dominated the elements of the middle ground. Even the stairs in the middle ground rarely drew fixations. The foreground elements were less attractive in the alternative with the vista.

\section{GENERAL OBSERVATIONS}

The following is a summary of some relevant positions that appear to emerge from the research. They are speculation at this point, and should all be analyzed further before conclusive lessons can be drawn.

- Generally, view fixations were not directed at the nonfurnished areas of the spaces, indicating that perceptual backgrounds are not visually attractive. One might speculate that the so-called negative space or perceptual background is only then attractive to the eye, if it possesses a clearly defined, regular shape, such as square or rectangular forms. This was the case, for example, with the negative space above the staircase in the symmetrically arranged altemative .

- Spatial contours are commonly not scanned by the eye. In the furnished alternatives, fixations were rarely directed toward the corners of spaces. Only in the example of the empty space did a few fixations occur in these areas.

- Rectilinearly oriented contours of objects were usually not scanned; only obliquely oriented objects were tracedfor example, when a set of stairs was shown from the side.

- In the case of vertically oriented spatial elements such as columns, only the lower and upper regions formed centers of attraction, with the upper part being slightly more attractive.

- The foreground is generally more attractive for initial fixations. During further fixations elements in the foreground also form stronger centers of attraction than elements in the middle ground or background.

\section{SUMMARY}

Research using the type of investigation deployed here is only in its fledgling stages. Therefore, one can only speculate as to the meaning of the above observations. Most interestingly, it appears that forms or objects which deviate from the Cartesian frame of horizontal and vertical reference seem to attract greater attention than those that do not. For example, Hubel and Wiese ${ }^{10}$ found several decades ago that there are particular groups of cells on the visual cortex which react only to horizontal and vertical stimuli. This may provide a clue to the reason why viewers needed to scan contours individually which departed from this spatial reference (for instance, the side elevation of the stairs).

The dominance of the left part of a space over the right is also a phenomenon that has been pointed out numerous times. For example, Arnheim has noted in several instances that the left side of paintings can afford greater visual weight than the right side. ${ }^{11}$ A great deal of investigation concerning this preference for the left is collected in the book Der Linksdrall in der Natur. This book primarily concerns a left preference of all organic growth processes, but it also describes an apparent preference of the left hemisphere in the perception of images and spaces. (A further note in this regard is that movie and theater directors often place single actors who are to dominate a scene, such as the singer of an aria, in the left front part of a space.)

It is, moreover, notable that the tests revealed few centers of fixation in the perceptual background. One could interpret this to mean that perceptual figures are visually more important than the background. This confirms the hypothesis of gestalt psychology that the figure dominates the ground. In relation to this observation, it must be pointed out that in these tests, even when the background took a regular form, such as a square or a rectangle, the visual attractivity of these areas was not notably strengthened by the regularity.

Finally it must be pointed out that for a symmetrical pattern the factor of redundancy seemed to play a role insofar as symmetrically arranged spatial elements were only investigated in detail once. This data then appeared to be sufficient for the perceptual processing of the symmetrically oriented element.

In summary, in relation to architectural issues one of the most important apparent findings of this initial research appears to be that the perception of architectural forms and spaces is not based on the scanning of individual stimuli, such as contours, but on forms as a whole. However, the results of this first test series do not suffice to allow clear statements about which precise geometric properties of form determine eye-movement sequences. The current results, nevertheless, support a theory of visual centers in architecture, as described for two-dimensional images by Arnheim, ${ }^{12}$ and proposed for spatial situations by Weber. ${ }^{13}$ Such a concept of visual or compositional centers is well known in the arts, but to this point it has rested on common sense rather than scientific evidence. The experiments described in this paper make the case for further testing using eye movement equipment. In time, more conclusive statements about the nature of perception in relation to architectural forms and spaces may emerge.

\section{NOTES}

' Arnheim, R., Art and Visual Perception. Berkeley and Los Angeles, 1954.

, The Dynamics of Architectural Form. Berkeley and Los Angeles, 1977.

2 Buswell, G.T. How People Look at Pictures, Chicago 1935

${ }^{3}$ Brandt, H.F. The Psychology of Seeing, New York 1945

${ }^{4}$ Kaufman and Richards, Spontaneous Fixation Tendencies for Visual Forms, Perception and Psychophysics. 1969 vol.5. pp. $85-88$.

5 Molnar, F. About the Role of Visual Exploration in Aesthetics, 1981

"Levey-Schoen, A, 1973, "Position of stimuli in the visual field and within a pattern, as determinants of the fixation response", in The Oculomotor System and Brain Function. Ed. V Zikmnd London. pp 243-255 
7 Gould J D, Peeples D R, "Eye movements during visual search and discrimination of meaningless, symbol and object patterns", J. Exp. Psychol 85, 51-55 (1970)

${ }^{8}$ Noton. D. and L. Stark. Eye Movement and Visual Perception. Scientific American 1971, vol. 244, pp. 34 - 53.

9 ibid.

${ }^{10}$ Hubel, D.H., and T.N. Wiesel. Brain Mechanisms of Vision. In: Atkinson ed., Psychology in Progress. New York, 1975.,s.3244.

"Arnheim, R., The Power of the Center. Berkeley and Los Angeles, 1985.

12 Arnheim, op.cit. ch.1.
13 Weber, R. Shapes of the Void - Figural Definitions of Architectural Space. Lecture at the American Society for Aesthetics, Portland 1992 and Weber, R. On the Aesthetics of Architecture - A Psychological Approach to the Structure and the Order of Perceived Architectural Space, London 1995

* This project was begun with a grant from the Graham Foundation for the Advancement of the Arts, Chicago. Further funding, especially for hardware and research assistants, was provided by the National Endowment for the Arts, Washington. The University of California, Berkeley, provided laboratories and student assistants. 